Pediatr. Res. 16: 1052-1053 (1982)

\title{
Subject Index to Volume 16
}

Abortion, spontaneous 1022

Abstracts, American Pediatric Society and Society for Pediatric Research, Annual Meeting 79A

Abstracts, European Society for Pediatric Endocrinology 886

Abstracts, European Society for Pediatric Gastroenterology and Nutrition 1037

Abstracts, European Society for Pediatric Research, Annual Meeting 688

Abstracts, Section of Rheumatology at American Academy of Pediatrics 166

Acid lipase deficiency 954

Acid, organic 771

Acrodermatitis enteropathica 599

Acute renal failure 277

Acyl-CoA dehydrogenation, multiple 861

Adenine nucleoside 362

Adenosine deaminase deficiency 362

Adenylate cyclase 381,463

Adipocytes 982

Adrenal gland 65, 172, 682

Adrenal hyperplasia 60

Adrenoceptors, alpha 340

Adrenoleukodystrophy 172

Aldosterone 354

Alpha $_{1}$-antitrypsin 192

Alpha $2_{2}$-macroglobulin 416, 613

Alpha-methylphenylalanine 388

Amiloride 1035

Amino acids 213, 324, 388, 751

Amikacin 810

Aminoglycosides 810

Amniocentesis 172

Amniotic fluid 109

Anemia 1016

Antibodies, secretory 272

Antibody-dependent cytotoxicity 558, 995

Antibody response 176

Antibiotic 509

Apnea 621

Apolipoprotein 665

Arginine 569

Arginine esterase 613

Asphxia 152, 156, 806

Astrocytes 187

Astroglial cells 187

Base deficit 156

Batten disease 530

Beta-adrenergic receptors 381,463 , 591

Beta cell 213

Beta-hydroxy-beta-methylglutarate 644
Beta-hydroxybutyrate 644

Beta-thalassemia 286

Bicarbonate 104, 533

Bile 282

Bile acid 494

Bile salt 69,251

Bilirubin 130, 670

Biotin 126

Blood-brain barrier 40, 167, 324

Blood coagulation 8,732

Blood flow, cerebral 499

Blood flow, uteroplacental 716

Blood gases 705

Blood pressure 163

Blood viscosity 964

Body composition 784

Body position 943

Bone age 343

Brain 187, 324, 455, 499, 520, 806

Brain blood flow 137

Brain damage 412

Brain metabolism 40, 579

Breast milk 89

Breathing, control 779

Bronchopulmonary dysplasia 290 , 446, 798, 969

Brush border membrane 441, 644

Caffeine 167

Calcemia 75

Calcitonin 75

Calcium 566, 675

Calf thymic hormone 573

Carbohydrate 1025

Cardiac output 594, 705

Carnitine 89, 877

Catecholamines 463, 632

Cerebrospinal fluid 751

Chemotactic activity 1000

Chemotactic factor inactivator 538

Cholecystokinin-octapeptide 123

Cholesterol 665, 682

Choline 324

Choline kinase 561

Chromosome aberration 663

Chromosome abnormality 343

Chromosome, y 769

Cirrhosis 192

Cockayne syndrome 92

Coeliac disease 441, 1004.

Colistin 509

Colon 295

Colon 776

Colostrum 272, 604

Colostrum 995

Concanavalin A 314, 441

Congenital dyserythropoietic anemia 1016

Cord serum 243

Corticosteroids 50

Creatine kinase 806

Cystathionase 65
Cystathionine 65

Cysteine 65

Cystic fibrosis $13,21,60,104,181$, $203,223,416,494,533,554$, $613,761,792,874,938,1035$

Cystine 86

Cystinosis 86, 213

Cytidine nucleotides 331

Cytidine triphosphate 331

Cytomegalovirus 192, 455

D-beta-Hydroxybutyrate 579

Diabetes 608, 632

Diazoxide therapy 1

1, 25-Dihydroxyvitamin $\mathrm{D}_{3} 75$

Diabetes 869

Diabetes mellitus, maternal 827

Diarrhea 329

Dicarboxylic aciduria 861, 877

Dipalmitoyl phosphatidylcholine 515

Depeptide transport 99

Diplegia 45

Disacchariflase 318

Dithiothreitol 86

Dolichols 530

Down Syndrome 314, 769

D-Penicillamine 370

Duram gliadin peptides 1004

Dwarfism, psychosocial 299

E. coli 243,329

EEG 30

Electrolyte 223

Encephalitis 176

Energy 1025

Enterocyte 441

Enzyme replacement 227

Epidemiology 82

Epidermal growth factor 520,820

Epinephrine 982

Erythrocyte 227, 331, 761, 928, 933, $960,964,1016$

Erythrocyte, transfusion 227

Esophageal sphincter 851

Essential fatty acid 599

Ethanolamine kinase 561

Euthanasia 846

Factor IX 256

Fatty acids 45, 69, 172, 761, 877, 950

Fetal activity 109

Fetal growth 109

Fibroblast 86, 232

Fick method 594

Folate enzymes 628

Gastrin 851

Gentamicin 509

Germinal matrix 499

Globin chain 1016
Glomerular filtration rate 351

Glomerulus 436

Glucagon 632, 1010

Glucagon, immunoreactive 1

Glucocorticoid 776, 827

Gluconeogenesis 632

Glucose 304, 310, 579, 608, 746, 776

Glutaric aciduria 877

Glutathione 377

Glycine 5

Glycinin 728

Glycogen 50, 869

Glycopeptide 203

Glycoproteins 176, 973

$\mathrm{GM}_{2}$ ganglioside 217,232

Gram-negative 509

Granulocyte 57,373

Group B streptococci 209, 347, 549, 816

Growth factors 820

Growth hormone 50, 299, 1010

Haemophilia B 256

Heart 357, 463, 721

Hemoglobin 286

Heparan sulfate 395

Heparin 181

Hepatitis B e antigen 247

Hepatocyte 282

Herpes simplex virus 176

Hexacosanoic acid 172

Hexosaminidase A 217, 232

Hexose monophosphate 377

Hippocampus 187

Homocysteine 370

Homocystine 370

Homocystinurics 370

Hormone-sensitive lipase 982

Hyaline membrane disease 35, 446

Hydrocortisone 123

Hydroxyl radical 856

1, 25-Hydroxyvitamin $\mathrm{D}_{3} 75$

Hyperbilirubinemia 130, 670

Hyperglycemia 653, 746

Hyperglycinemia 5

Hyperinsulinemia 632

Hyperphenylalaninemia 388

Hyperthyroidism 238

Hyperviscosity 148

Hypoglycemia 40, 632, 877

Hypoglycemia, leucine-sensitive 1

Hypophysectomy 561

Hypotension 137

Hypothyroidism 238, 381, 463, 570

Hypoxemia 8, 732, 653

Hypoxia 30, 152, 156, 705

Hypoxanthine 152, 156

Ichthyosis 45

IgA 272

$\operatorname{IgD} 604$

IgE 604 
$\operatorname{lgG} 181,247$

Ileum 295, 494, 756, 776,

Iminoglycinuria 684

Iminopeptide 227

Immune response, neonatal 649

Immunodeficiencies 573

Immunoglobulin 649

Immunoreactive glucagon 1

Immunoreactive insulin 1

Indomethacin 599

Insulin 85, 820, 1010

Interstitial lung disease 779

Intestine 99, 295, 318, 728, 756, 948, 1004

Intraventricular hemorrhage 499

Iron, deficiency 318

Isocaloric feeding 141

Isoelectric focusing 13,21

Isoleucine 751

Isomaltase 948

Isovaline acidemia 771

Jejunum 295, 728, 756, 776

Kallekrein 613

Kidney $65,148,351,436,644,684$, $784,840,973$

Kwashiorkor 663

Lactase 318

Lactate 156,324

Lactation 113

Lactiacidemia 85

Lactic acid 40

Lactic acidosis 85

Lactose 60, 566

Laryngeal chemoreflex 621

Lead poisoning 331

Leukocyte 232, 243, 373

Lipase 69, 882

Lipid 45, 251, 665, 882, 1025

Lipolysis 251, 982

Lipoprotein 665,682

Lipoteichoic acid 816

Liver 5, 65, 192, 282, 377, 395, 628, $632,950,977$

Lung 50,130, 209, 238, 290, 381, 446, 561, 570, 583, 711, 779, $798,943,969$

Lymphocyte 314, 649

Lysozyme 874

Macrophages 209, 711

Macrosomia 653

Magnesium 295, 675

Malformations, congenital 973

Malnutrition 187, 1010

Maltase 318

Mean airway pressure 35

Mecuric chloride 227

Methylmalonic acidemia 740

Microspheres 148, 594
Milk 113, 251, 659

Milk, human 882

Milk, neonatal 60

Mineralocorticoid 118

Monoclonal antibodies 938

Mononuclear cells 558

Moral reasoning 846

Mucin 792

Mucociliary inhibition 13, 21

Mucopolysaccharide, acid 395

Mucosal cells 494

Multiple carboxylase deficiency 126

Multiple sulfatase deficiency 395

Muscle 335

Myocardium 340, 357, 463

Natriuresis 840

Natural killer cytotoxicity 558

Necrotizing enterocolitis 82,509

Nephrons 684

Nephrotoxicity 810

Nerve growth factor 520

Neuroblastoma 199

Neuroendocrine cells 446

Neuromuscular diseases 335

Neuronal ceroid lipofuscinosis 530

Neurohypophysis 569

Neutral lipid storage 954

Neutropenia 57

Neutrophil 373, 525, 549, 591, 856

Nitrogen 113, 659

Nucleotide 659

Nutrition 251, 304, 784

Nyctohemeral rhythm 354

Oleic acid 579

Oligosaccharide 304

Ontogeny 820

Opsonization 243

Ornithine decarboxylase 721

Ornithine transcarbamylase deficiency 977

Osmolar load 141

Ouabain 357

Oxidative metabolism 373,525

Oxygen consumption 243,705

Oxygen toxicity 583

Pain 569

Pancreas 65, 104, 123, 213, 554, 948, 1010

Parathormone 75

Parenteral alimentation 304

Peroxisome 977

pH 156

Phagocytes, colostral 995

Pharmacokinetics 810

Phenylketonuria 213, 388, 751

Phosphate 675

Phosphoenolpyruvate carboxykinase 632
Phosphofructokinase 199

Phospholipid 45, 130, 515, 761, 834

Phosphorus 75

Phosphorylase 50

Phototherapy 670

Placenta, human 820

Platelets 1000

Pneumonia 209

Polymorphonuclear leukocyte 558

Polyamine 314,721

Polycythemia 148

Polypeptides 176

Potassium 776

Power spectral analysis 30

Prenatal diagnosis 172, 256, 740

Prolidase 227

Proline 684

Prostaglandin E 599

Prostaglandin $\mathrm{F}_{\mathrm{lk}} 827$

Protease 69, 948

Protein 60

Protein deficiency 784

Proteinase-binding 416

Pulmonary mechanics 290

Putrescine 314, 721

Pyrimidine 5'-nucleotides 331

Pyruvate kinase 199

Pyruvic acid 85

Radioimmunoassay 247

REM 512

Renal failure 784

Renal hypertrophy 436

Renal oxygen consumption 351

Renin 118, 354

Reserpine 104

Respiration 1031

Respiratory distress syndrome 35 , $515,827,834,969$

Reye's syndrome 861,950

Rhabdomyosarcoma 199

Riboflavin 861

Rotavirus 329

Salt-losing congenital adrenal hyperplasia 118

S. aureus 57,243

S. cerevisiae 243

S. faecalis 243

Secretagogues 123

Secretin 104

Senior syndrome 954

Sister chromatid exchange 663

Sjogren-Larsson syndrome 45

Skeletal maturation 343

Skinfold thickness 989

Sleep 299, 512, 621

Sodium 113, 118, 277, 351, 776

Soyasaponins 728

Soybean lectin 728

Spermidine 314, 721

Spermine 314,721
Spinal fluid 412

Stem cell 549

Steroid 682

Stomach 141

Streptozotocin 869

Submandibular gland 223

Submaxillary gland 520

Sucrase 318, 948

Sudden infant death syndrome 130

Superoxide 373

Superoxide dismutase 570, 933, 1000

Surfactant 130, 515, 711, 834

Sweat glands 533

Taurocholate 282

Tay-Sachs disease 217, 232

T cell defect 573

Teratogen 973

Teratoma 199

Tetraplegia 45

Theophylline 167, 649, 982

Therapy, prenatal 126

Thermodilution 594

Thermoregulation 512

Thoracotomy 705

Thymic hormone 92

Thyroid hormones 520

Thyroxine $377,381,570$

Trachea 830

Transferrin C 1022

Trigeminal stimulation 1031

Triglyceride 60

Triiodothyronine 238

Triosephosphate isomerase 960

Trisomy 21769

Tyrosinosis 213

Umbilical cord 109

Undernutrition 272

Uranyl nitrate 277

Urine 21,771

Valine 751,756

Valentia G 1215

Vascular resistance 163

Vasopressin 569

Ventricle 463, 721

Virus killing 525

Viscera 455

Vitamin E 583, 928

Water, extracellular 989

Wheat gliadin peptides 1004

Witch's milk 460

Wolman's disease 954

Yellow hyaline membrane disease 130

Zinc 566, 599 